\title{
Primary pineal rhabdomyosarcoma successfully treated by high-dose chemotherapy followed by autologous peripheral blood stem cell transplantation: case report
}

\author{
Yukitomo Ishi, MD, ${ }^{1}$ Shigeru Yamaguchi, MD, ${ }^{1}$ Akihiro Iguchi, MD, ${ }^{2}$ Yuko Cho, MD, ${ }^{2}$ \\ Junjiro Ohshima, MD, ${ }^{2}$ Kanako C. Hatanaka, MD, ${ }^{3}$ Emi Takakuwa, MD, ${ }^{3}$ Hiroyuki Kobayashi, MD, ${ }^{1}$ \\ Shunsuke Terasaka, MD, ${ }^{1}$ and Kiyohiro Houkin, MD'1
}

Departments of ${ }^{1}$ Neurosurgery and ${ }^{2}$ Pediatrics, Hokkaido University Graduate School of Medicine; and ${ }^{3}$ Department of Surgical Pathology, Hokkaido University Hospital, Sapporo, Hokkaido, Japan

\begin{abstract}
Primary intracranial rhabdomyosarcoma is quite rare, and its prognosis is poor compared with that for rhabdomyosarcoma in other organs. The authors present a case of pineal rhabdomyosarcoma successfully managed with multimodal therapy including surgery, chemotherapy, radiation, and high-dose chemotherapy (HDC) followed by autologous peripheral blood stem cell transplantation (HDC/APBSCT). An 8-year-old girl presenting with headache and nausea was referred to the authors' institution. Computed tomography and MRI revealed a pineal tumor associated with obstructive hydrocephalus. Subsequently, an emergent endoscopic tumor biopsy and third ventriculostomy were performed. The patient's symptoms immediately improved. The most likely pathological diagnosis was embryonal rhabdomyosarcoma. Chemotherapy with etoposide, cyclophosphamide, cisplatin, pirarubicin, ifosfamide, actinomycin $\mathrm{D}$, and vincristine was followed by a second-look operation and whole-brain and craniospinal radiation. Because the intraoperative findings and pathological examination of the second operation suggested a definitive diagnosis of rhabdomyosarcoma and the presence of viable residual tumor cells, HDC with etoposide and melphalan was followed by APBSCT. The patient was discharged from the hospital without residual tumor or any neurological deficit. No recurrence was observed at 30 months. This is the first case of primary pineal rhabdomyosarcoma treated with HDC/APBSCT. Although the efficacy of HDC/APBSCT for rhabdomyosarcoma has not been established, the prognosis of primary intracranial rhabdomyosarcoma treated with conventional treatment is quite poor. High-dose chemotherapy followed by APBSCT may contribute to a better prognosis for primary intracranial rhabdomyosarcoma.
\end{abstract}

http://thejns.org/doi/abs/10.3171/2015.12.PEDS15419

KEY WORDS rhabdomyosarcoma; brain tumor; pineal tumor; high-dose chemotherapy; blood stem cell transplantation; oncology

$\mathrm{R}$ HABDOMYOSARCOMA is the most common soft-tissue sarcoma that occurs in childhood and adolescence. ${ }^{3}$ This sarcoma originates from skeletal muscle and is divided into 2 major subtypes according to its pathology, genetics, clinical presentation, and outcome: embryonal and alveolar. ${ }^{20}$ Although $16 \%-40 \%$ of rhabdomyosarcomas arise in the head and neck region, most occur in the nasal cavity, parameningeal site, or orbit. ${ }^{2,3,7,20}$ Primary intracranial rhabdomyosarcoma is extremely rare, and only 48 cases have been reported..$^{8,18}$ Most, however, were reported in the 20th century, and few patients underwent intensive treatment with chemotherapy and radiotherapy. Therefore, the efficacy of chemotherapy and radiotherapy for primary intracranial rhabdomyosarcoma is unknown, and the treatment strategy has not been established. We present a case of primary rhabdomyosarcoma arising in the pineal region that was successfully managed with aggressive multimodal therapy including surgery, chemo-

ABBREVIATIONS APBSCT = autologous peripheral blood stem cell transplantation; $\mathrm{CBDCA}=$ carboplatin; $\mathrm{CDDP}=$ cisplatin; $\mathrm{CPA}=$ cyclophosphamide; $\mathrm{CR}=$ complete remission; GCT = germ cell tumor; HDC = high-dose chemotherapy; IRS-V = Intergroup Rhabdomyosarcoma Study V; L-PAM = melphalan; SLO = second-look operation; VP-16 = etoposide.

SUBMITTED July 8, 2015. ACCEPTED December 17, 2015.

INCLUDE WHEN CITING Published online March 4, 2016; DOI: 10.3171/2015.12.PEDS15419. 
therapy, radiation, and high-dose chemotherapy (HDC) followed by autologous peripheral blood stem cell transplantation (HDC/APBSCT).

\section{Case Report}

History, Examination, and First Operation

An 8-year-old girl complaining of headache and nausea visited another hospital. A pineal mass was noted on head CT, and the patient was referred to our institution. An admission neurological examination revealed no significant deterioration; however, she did complain of headache. Head CT (Fig. 1A and B) revealed dilation of the bilateral ventricles and a pineal mass without calcification or hemorrhage. Magnetic resonance imaging (Fig. 1C-F) revealed a pineal mass $35 \mathrm{~mm}$ in diameter that showed hypointensity on T1-weighted imaging, hyperintensity on T2-weighted imaging, and good enhancement on contrastenhanced MRI using gadolinium. $\beta$-Human chorionic gonadotropin and $\alpha$-fetoprotein were negative in serum and cerebrospinal fluid. We performed emergent endoscopic third ventriculostomy for obstructive hydrocephalus and tumor biopsy. A flexible neuroendoscope (VISERA ventricular videoscope, type VEF-V, Olympus) was inserted from the right frontal lobe with an approach to the anterior horn of the right lateral ventricle. The patient's symptoms improved soon after the surgery.

\section{Pathological Examination}

A small biopsy specimen was obtained for pathological analysis. Hematoxylin and eosin staining (Fig. 2A and B) revealed a bundle-like proliferation of hyperchromatic, round, and spindle-shaped immature cells and spindle cells with eosinophilic cytoplasm with differentiated myofibrils resembling striated muscle cells. These tumor cells accounted for almost the entire specimen. Cartilage components were also observed but were only found in a small portion of the specimen. Components suggestive of germ cell tumor (GCT) or pineoblastoma were not observed. On immunohistochemical staining, striated muscle-like tumor cells stained positive for desmin (Fig. 2C), and immature tumor cells were also partly positive for desmin. The labeling index for Ki-67 was significantly high, with $>50 \%$ Ki-67-positive tumor cells (Fig. 2D). In addition, negative placental alkaline phosphatase (PLAP), oct 3/4, synaptophysin, chromogranin A, and neurofilaments in any of the tumor cells also supported the lack of evidence for GCT and pineoblastoma. The most likely pathological diagnosis was embryonal rhabdomyosarcoma.

\section{Second-Look Operation and Postoperative Treatment}

The tumor was growing rapidly approximately 2 weeks after the operation (Fig. 3A). Contrast-enhanced wholebody CT revealed no apparent metastatic lesions, and the pretreatment staging ${ }^{10}$ was estimated as Stage II. We administered 2 courses of chemotherapy with etoposide (VP-16; $100 \mathrm{mg} / \mathrm{m}^{2} /$ day on Days $1-5$ ), cyclophosphamide (CPA; $1200 \mathrm{mg} / \mathrm{m}^{2} /$ day on Day 2), and cisplatin (CDDP; $20 \mathrm{mg} / \mathrm{m}^{2} /$ day on Days $1-5$ ). An MRI study after 2 courses of chemotherapy (Fig. 3B) showed significant reduction of the tumor. During this period, to determine whether further applications of chemotherapy and radiotherapy were required, definitive histopathological diagnosis with a sufficient amount of tumor specimen was desirable. In addition, the patient's clinical condition was favorable for surgical intervention.
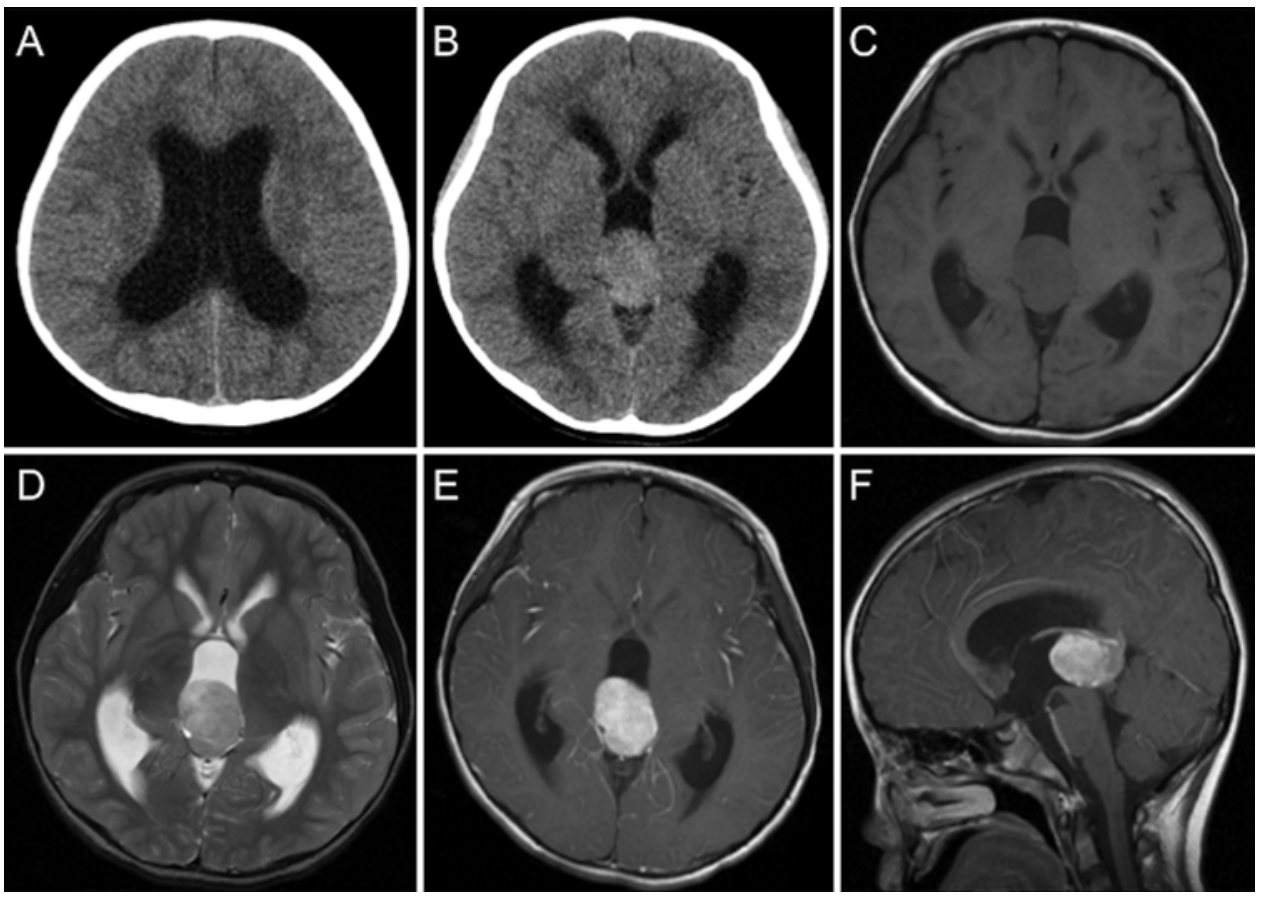

FIG. 1. Admission CT showed dilation of bilateral ventricles (A) and a pineal mass lesion without calcification or hemorrhage (B). On MRI, the pineal mass presented hyperintensity on T2-weighted sequences (C), hypointensity on T1-weighted sequences (D), and good enhancement on axial $(\mathbf{E})$ and sagittal $(\mathbf{F})$ contrast-enhanced MRI using gadolinium. 


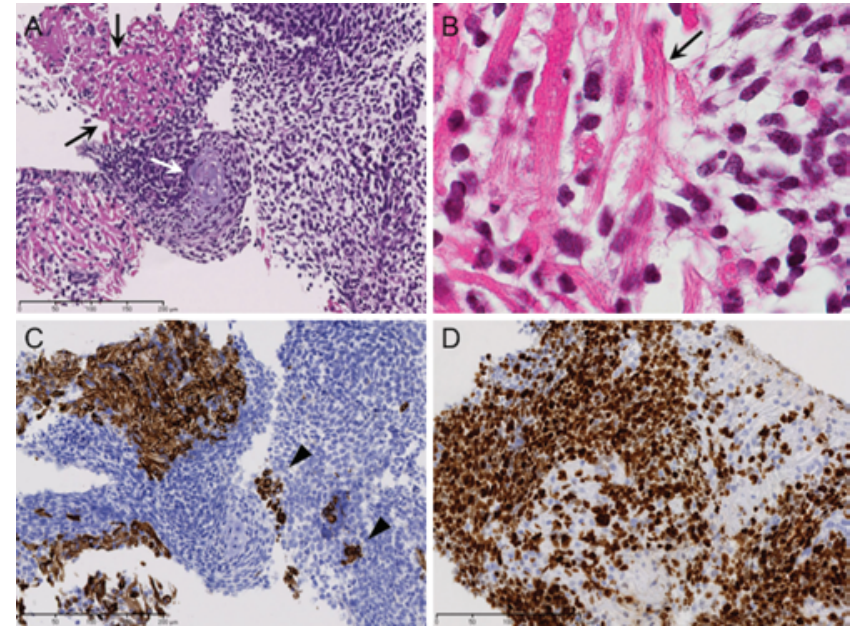

FIG. 2. Staining of the tumor (A and $\mathbf{B}$ ) showing bundle-like proliferation of small tumor cells with a spindle or round shape that are rich in chromatin, as well as tumor cells with eosinophilic cytoplasm that are similar to striated muscle cells (black arrows). Cartilage components are also visible (white arrow). Striated muscle-like tumor cells are positive for desmin, and parts of the immature tumor cells (arrowheads) are positive for desmin (C). The tumor cells show a high Ki-67 labeling index (D). H \& $E(A$ and $B)$ and desmin $(C)$, original magnification $\times 1000(B)$.

Therefore, we performed a second-look operation (SLO) via the left occipital transtentorial approach and resected the tumor as much as possible. Although we were able to resect a majority of the tumor, a small amount of residual tumor adhered firmly to the dorsal midbrain and could not be resected. At this operation, pathological examination (Fig. 4) of the tumor showed viable and striated muscle-differentiated tumor cells that were positive for desmin. These cells were therefore considered to be rhabdomyoblasts. Degenerative tissue formed by chemotherapy was also observed; however, immature tumor cells that were recognized in the first operation were not seen. In addition, other components suggestive of teratoma, GCT, or pineoblastoma were not observed, and a definitive diagnosis of rhabdomyosarcoma was confirmed.

Because MRI after the first operation (Fig. 3C) had also suggested the presence of a small amount of residual tumor, we administered further intensive chemotherapy on the basis of the radiological, intraoperative, and pathological findings. Therefore, after the SLO, the patient underwent 1 course of chemotherapy with VP-16 $\left(100 \mathrm{mg} / \mathrm{m}^{2} /\right.$ day on Days 1-5), CPA (1200 mg/m²/day on Day 2), and CDDP $\left(20 \mathrm{mg} / \mathrm{m}^{2} /\right.$ day on Days $\left.1-5\right)$; 1 course of chemotherapy with ifosfamide $\left(1800 \mathrm{mg} / \mathrm{m}^{2} /\right.$ day on Days $\left.1-5\right)$, VP-16 $\left(100 \mathrm{mg} / \mathrm{m}^{2} /\right.$ day on Days $\left.1-5\right)$, actinomycin D $(0.015$ $\mathrm{mg} / \mathrm{kg} /$ day on Days $1-5)$, and vincristine $\left(1.5 \mathrm{mg} / \mathrm{m}^{2} /\right.$ day on Days 8-15); 1 course of chemotherapy with VP-16 (100 $\mathrm{mg} / \mathrm{m}^{2} /$ day on Days $\left.1-5\right)$, CPA (1200 mg/m²/day on Day 2$)$, pirarubicin $\left(60 \mathrm{mg} / \mathrm{m}^{2} /\right.$ day on Day 3), and CDDP (20 mg/ $\mathrm{m}^{2} /$ day on Days 1-5); and focal radiation of 22.8 Gy and craniospinal radiation of $25.2 \mathrm{~Gy}$. Fourteen days after the last of the chemotherapy, peripheral blood stem cells were extracted. Subsequently, we performed HDC with VP-16 $\left(200 \mathrm{mg} / \mathrm{m}^{2} /\right.$ day on Day -8 to Day -4$)$ and melphalan (L-PAM; $90 \mathrm{mg} / \mathrm{m}^{2}$ /day on Days -3 and -2 ) followed by APBSCT (on Day 0). We referred to the high-dose L-PAM,
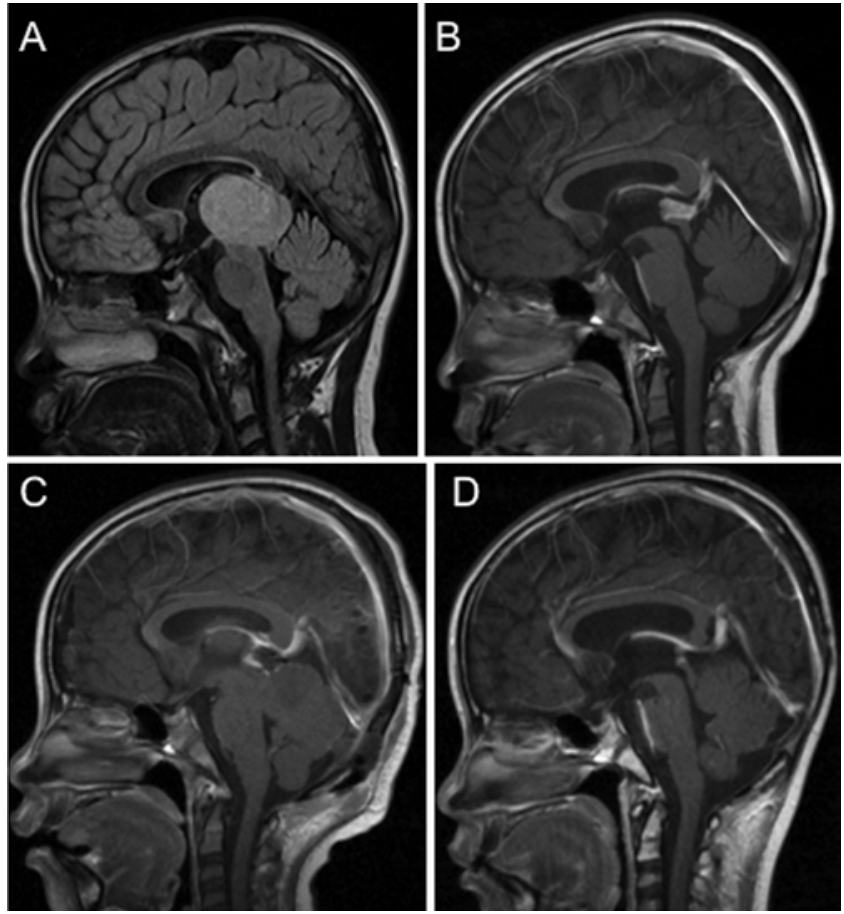

FIG. 3. Compared with the MRI studies obtained on admission, the MRI performed 13 days after the first operation $(\mathbf{A})$ showed significant tumor growth. The MRI performed after the second of 2 courses of chemotherapy with VP-16, CPA, and CDDP (B) showed significant tumor reduction. Postoperative MRI (C) suggested the presence of a small amount of residual tumor. The MRI studies after all treatments (D), including APBSCT, showed no evidence of recurrence.

VP-16, and carboplatin (CBDCA) (Hi-MEC) protocol ${ }^{11}$ for treatment but decided that the combined administration of CBDCA and L-PAM would present a risk because the patient's serum creatinine was elevated from 0.29 to $0.50 \mathrm{mg} /$ $\mathrm{dl}$ after the chemotherapy and radiotherapy. Therefore, we used VP-16 and L-PAM but not the CBDCA as the preparation regimen for APBSCT. For the APBSCT, 5.0 $\times 10^{6} \%$ $\mathrm{kg} \mathrm{CD} 34$-positive cells were infused, and the percentage of viable cells was $78 \%$. Mucosal damage appeared on Day 3 , and transient fever $>38^{\circ} \mathrm{C}$ appeared on Day 4, but blood culture detected no growth of bacteria. Serum neutrophil cells recovered to $>500 / \mu$ l on Day 10. After these treatments, MRI (Fig. 3D) showed no evidence of residual tumor. The patient was discharged from our hospital without any neurological deficit and was alive 30 months after diagnosis with no evidence of tumor.

\section{Discussion}

Among the previous 48 reports of primary intracranial rhabdomyosarcoma, the frontal lobe and cerebellum were the most common tumor sites. ${ }^{8,18}$ There has been no report of primary pineal rhabdomyosarcoma, but 2 cases of secondary rhabdomyosarcoma arising in a pineal teratoma have been described..$^{5,15}$ In the present case, mixed GCT or malignant transformation of a teratoma associated with a rhabdomyosarcomatous component was strongly considered as the differential diagnosis after the first operation. However, because no components apart from the rhabdomyosarcoma were observed, the patient was started on 


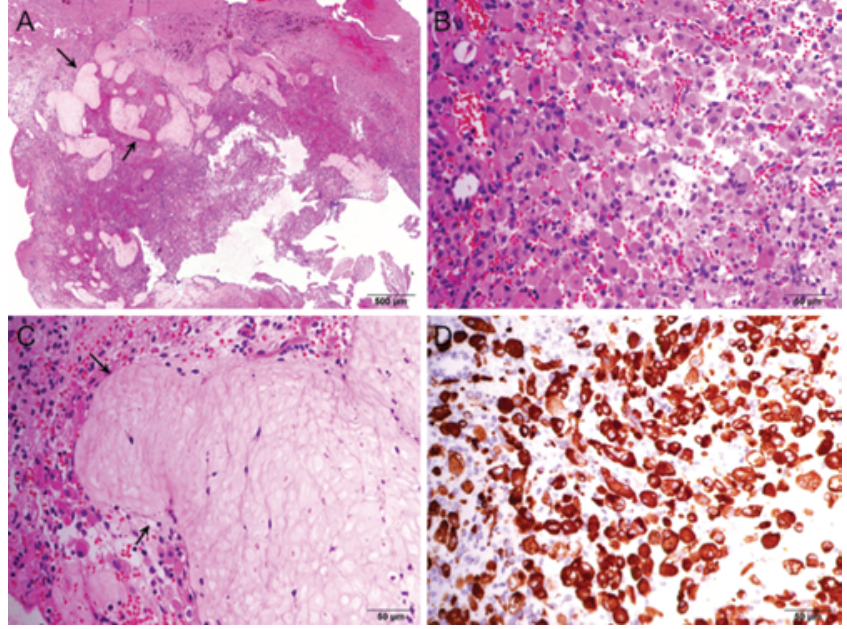

FIG. 4. Pathological examination of tumor resected at the second operation. Viable tumor cells with eosinophilic cytoplasm were similar to those observed in the first operation (A and $\mathbf{B}$ ). These tumor cells are positive for desmin (D). Degenerative tissue (black arrows, $\mathbf{A}$ and $\mathbf{C}$ ) suggests a treatment effect of chemotherapy. $H \& E(A-C)$, desmin (D).

treatment under the most likely diagnosis of rhabdomyosarcoma. Pathological examination at the second operation presented only components of striated muscle differentiation, and other tumor components were not observed. Therefore, a definitive diagnosis of rhabdomyosarcoma was eventually confirmed.

The prognosis of pediatric rhabdomyosarcomas has improved over the past 2 decades, and the 5-year survival rate is $>85 \%$ in patients with low-grade, localized embryonal rhabdomyosarcomas. ${ }^{20}$ However, the prognosis of metastatic rhabdomyosarcoma has not improved, and the 5 -year overall survival remains between $20 \%$ and $30 \%{ }^{3}$ Similarly, primary intracranial rhabdomyosarcoma has a poor prognosis, with overall survival reaching only 9.1 months. ${ }^{6}$ However, most reports of primary intracranial rhabdomyosarcoma were published in the 20th century, and very few patients in those reports underwent multimodal chemotherapy and radiotherapy. There are 8 previous cases (Table 1) of primary intracranial rhabdomyosarcoma with long-term survival $>24$ months. ${ }^{1,4,6,8,12,13,19}$ Postoperative radiation and chemotherapy with multiple anticancer drugs were administered in all but 1 patient, who received only radiation postoperatively. The treatment strategy has not been established because this tumor is too rare to conduct clinical study for treatment; however, results in these documented cases suggest the efficacy of multimodal treatment for primary intracranial rhabdomyosarcoma. In our case, the first 3 courses of chemotherapy with VP-16, CPA, and CDDP resulted in significant reduction of the tumor, which also indicated the efficacy of intensive chemotherapy using a multiagent regimen for primary intracranial rhabdomyosarcomas.

Second-look operation has been recommended in some reports, ${ }^{3,16}$ if feasible, for cases in which the tumor was not completely resected in the first operation. A lower relapse rate has been reported in patients who had complete remission (CR) with SLO than in those who obtained CR with chemotherapy alone. ${ }^{3}$ The existence of viable tumor in an SLO has also been reported as a risk factor for local control of rhabdomyosarcoma. ${ }^{16}$ Although the timing of an SLO is not standardized, as in our case, a previous protocol of the European Intergroup Study ${ }^{3}$ defined the period after the second course of chemotherapy as the appropriate time for an SLO. In our case, pathological examination after the SLO was useful to confirm viable tumor cells and chemotherapeutic efficacy.

The efficacy of HDC/APBSCT for rhabdomyosarcoma is controversial. Some authors have stated that a better metastatic rhabdomyosarcoma prognosis can be achieved with HDC/APBSCT treatment, ${ }^{7,9}$ but statistically proven efficacy has not been reported. ${ }^{14}$ Because of the poor prognosis of rhabdomyosarcoma in patients with advanced stage or metastatic disease, HDC/APBSCT has been performed experimentally in patients who were likely to have a poor response to the usual-dose chemotherapy. In pre-

TABLE 1. Summary of cases of primary intracranial rhabdomyosarcoma with long-term survival > 24 months

\begin{tabular}{|c|c|c|c|c|c|c|c|}
\hline Authors \& Year & $\begin{array}{c}\text { Age } \\
\text { (yrs), } \\
\text { Sex }\end{array}$ & Lesion Location & $\begin{array}{l}\text { Extent of } \\
\text { Resection }\end{array}$ & $\begin{array}{l}\text { Histological } \\
\text { Subtype }\end{array}$ & Chemotherapy & Radiotherapy & $\begin{array}{l}\text { Survival } \\
\text { (mos) }\end{array}$ \\
\hline Matsukado et al., 1975 & $3, \mathrm{~F}$ & 4 th ventricle & Subtotal & NA & - & 40 Gy local & $26, \mathrm{D}$ \\
\hline Shin \& Whitehead, 1980 & $9, \mathrm{M}$ & Cerebellar & Subtotal & NA & VAC/MTX & 15 Gy local, 30 Gy WB & $25, \mathrm{~A}$ \\
\hline Olson et al., 1985 & $3, \mathrm{~F}$ & Cerebellar & Subtotal & NA & VAC/MTX/Ara-C & $\begin{array}{l}14 \text { Gy local, } 30 \text { Gy WB, } \\
30 \text { Gy spinal }\end{array}$ & $67, \mathrm{~A}$ \\
\hline Celli et al., 1998 & $46, M$ & $\begin{array}{l}\text { Frontoparietal } \\
\text { lobe }\end{array}$ & Total & Embryonal & CYVADIC-VRC/CBDCA & 60 Gy local & $30, A$ \\
\hline Al-Gahtany et al., 2003 & $6, \mathrm{M}$ & Posterior fossa & Subtotal & NA & ACD/ADR/CPA/VCR & 50 Gy CS & $168, A$ \\
\hline Guilcher et al., 2008 & $5, \mathrm{~F}$ & Occipital lobe & Total & Embryonal & VICE-VAC & 45 Gy local & $26, \mathrm{~A}$ \\
\hline \multirow[t]{2}{*}{ Khalatbari et al., 2013} & $15, M$ & Frontal lobe & Total & Alveolar & VRC/ACD/ADR/IFM & $54 \mathrm{~Gy}$ & $47, \mathrm{D}$ \\
\hline & $3, \mathrm{M}$ & Frontal lobe & Total & Alveolar & VRC/ACD/ADR/IFM & $45 \mathrm{~Gy}$ & $36, \mathrm{~A}$ \\
\hline Present case & $8, F$ & Pineal region & Total & Embryonal & $\begin{array}{l}\text { IFM/VP-16/ACD/VCR, VP-16/CPA/ } \\
\text { THP/CDDP, L-PAM/VP-16 }\end{array}$ & $\begin{array}{l}22.8 \text { Gy local, } 25.2 \\
\text { Gy CS }\end{array}$ & $30, \mathrm{~A}$ \\
\hline
\end{tabular}

$A=$ alive; $A C D=$ actinomycin $D ; A D R=$ doxorubicin; $A r a-C=$ cytarabine $; C S=$ craniospinal $; C Y V A D I C=$ cyclophosphamide, doxorubicin, vincristine, and dacarbazine; $\mathrm{D}=$ dead; IFM = ifosfamide; $M T X=$ methotrexate; $N A=$ not available; $T H P=$ pirarubicin; $V A C=$ vincristine, $A C D$, and CPA; VICE = vincristine, ifosfamide, $C B D C A$, and VP-16; VRC = vincristine; $W B=$ whole brain. 
vious reports, Hosoi et al. ${ }^{7}$ demonstrated the efficacy of HDC/APBSCT for a high-risk group (patients with metastatic embryonal type who were older than 10 years and all patients with the metastatic alveolar type classified in the Intergroup Rhabdomyosarcoma Study V [IRS-V]). ${ }^{17}$ Although our case was not actually classified as high risk in the IRS-V, we applied whole-brain and spinal radiation and HDC/APBSCT in addition to conventional chemotherapy after considering the poor prognosis of primary intracranial rhabdomyosarcoma, the presence of residual tumor in the intraoperative findings at the SLO, and the existence of viable tumor cells in the pathological examination after the SLO. This is the first case of primary intracranial rhabdomyosarcoma treated using HDC/APBSCT in which the patient has survived for $>24$ months. Studies with longer follow-up periods and a greater number of cases are needed to prove the superiority of HDC/APBSCT versus conventional chemotherapy and radiation.

\section{Conclusions}

This is the first reported case of primary pineal rhabdomyosarcoma and the first case of primary intracranial rhabdomyosarcoma successfully managed with multimodal treatment including surgery, chemotherapy, radiation, and HDC/APBSCT. The adequacy of HDC/APBSCT is still controversial for high-risk rhabdomyosarcomas; hence, more cases are required to certify the efficacy of this treatment for primary intracranial rhabdomyosarcomas.

\section{References}

1. Al-Gahtany M, Shroff M, Bouffet E, Dirks P, Drake J, Humphreys R, et al: Primary central nervous system sarcomas in children: clinical, radiological, and pathological features. Childs Nerv Syst 19:808-817, 2003

2. Breneman JC, Lyden E, Pappo AS, Link MP, Anderson JR, Parham DM, et al: Prognostic factors and clinical outcomes in children and adolescents with metastatic rhabdomyosarcoma-a report from the Intergroup Rhabdomyosarcoma Study IV. J Clin Oncol 21:78-84, 2003

3. Carli M, Colombatti R, Oberlin O, Bisogno G, Treuner J, Koscielniak E, et al: European intergroup studies (MMT4-89 and MMT4-91) on childhood metastatic rhabdomyosarcoma: final results and analysis of prognostic factors. J Clin Oncol 22:4787-4794, 2004

4. Celli P, Cervoni L, Maraglino C: Primary rhabdomyosarcoma of the brain: observations on a case with clinical and radiological evidence of cure. J Neurooncol 36:259-267, 1998

5. Glass RL, Culbertson CG: Teratoma of pineal gland with choriocarcinoma and rhabdomyosarcoma. Arch Pathol (Chic) 41:552-555, 1946

6. Guilcher GM, Hendson G, Goddard K, Steinbok P, Bond M: Successful treatment of a child with a primary intracranial rhabdomyosarcoma with chemotherapy and radiation therapy. J Neurooncol 86:79-82, 2008

7. Hosoi H, Teramukai S, Matsumoto Y, Tsuchiya K, Iehara T, Hara J, et al: A review of 331 rhabdomyosarcoma cases in patients treated between 1991 and 2002 in Japan. Int J Clin Oncol 12:137-145, 2007

8. Khalatbari MR, Hamidi M, Moharamzad Y: Primary alveolar rhabdomyosarcoma of the brain with long-term survival. J Neurooncol 115:131-133, 2013
9. Kim NK, Kim HS, Suh CO, Kim HO, Lyu CJ: Clinical results of high-dose chemotherapy followed by autologous peripheral blood stem cell transplantation in children with advanced stage rhabdomyosarcoma. J Korean Med Sci 27:1066-1072, 2012

10. Lawrence W Jr, Anderson JR, Gehan EA, Maurer H: Pretreatment TNM staging of childhood rhabdomyosarcoma: a report of the Intergroup Rhabdomyosarcoma Study Group. Cancer 80:1165-1170, 1997

11. Matsubara H, Makimoto A, Higa T, Kawamoto H, Takayama J, Ohira M, et al: Possible benefits of high-dose chemotherapy as intensive consolidation in patients with highrisk rhabdomyosarcoma who achieve complete remission with conventional chemotherapy. Pediatr Hematol Oncol 20:201-210, 2003

12. Matsukado Y, Yokota A, Marubayashi T: Rhabdomyosarcoma of the brain. J Neurosurg 43:215-221, 1975

13. Olson JJ, Menezes AH, Godersky JC, Lobosky JM, Hart M: Primary intracranial rhabdomyosarcoma. Neurosurgery 17:25-34, 1985

14. Peinemann F, Kröger N, Bartel C, Grouven U, Pittler M, Erttmann R, et al: High-dose chemotherapy followed by autologous stem cell transplantation for metastatic rhabdomyosarcoma-a systematic review. PLoS One 6:e17127, 2011

15. Preissig SH, Smith MT, Huntington HW: Rhabdomyosarcoma arising in a pineal teratoma. Cancer 44:281-284, 1979

16. Raney B, Stoner J, Anderson J, Andrassy R, Arndt C, Brown $\mathrm{K}$, et al: Impact of tumor viability at second-look procedures performed before completing treatment on the Intergroup Rhabdomyosarcoma Study Group protocol IRS-IV, 19911997: a report from the children's oncology group. J Pediatr Surg 45:2160-2168, 2010

17. Raney RB, Anderson JR, Barr FG, Donaldson SS, Pappo AS, Qualman SJ, et al: Rhabdomyosarcoma and undifferentiated sarcoma in the first two decades of life: a selective review of intergroup rhabdomyosarcoma study group experience and rationale for Intergroup Rhabdomyosarcoma Study V. J Pediatr Hematol Oncol 23:215-220, 2001

18. Rivero-Garvía M, Márquez-Rivas J, Rivas E, Medina-López D, Quiroga-Cantero E: Primary cerebral radiotherapyinduced rhabdomyosarcoma: treatment with intraoperative carmustine implants. Pediatr Hematol Oncol 30:1-3, 2013

19. Shin KH, Whitehead VM: Rhabdomyosarcoma of the brain. Can J Surg 23:576-578, 1980

20. Wang C: Childhood rhabdomyosarcoma: recent advances and prospective views. J Dent Res 91:341-350, 2012

\section{Disclosures}

The authors report no conflict of interest concerning the materials or methods used in this study or the findings specified in this paper.

\section{Author Contributions}

Conception and design: Ishi. Acquisition of data: Yamaguchi, Iguchi, Cho, Ohshima, Hatanaka, Takakuwa, Kobayashi, Terasaka. Analysis and interpretation of data: Ishi, Hatanaka, Takakuwa. Drafting the article: Ishi. Critically revising the article: Yamaguchi, Hatanaka, Iguchi, Cho. Reviewed submitted version of manuscript: Yamaguchi. Administrative/technical/ material support: Yamaguchi, Iguchi, Cho, Ohshima, Kobayashi, Terasaka. Study supervision: Terasaka, Houkin.

\section{Correspondence}

Yukitomo Ishi, Department of Neurosurgery, Hokkaido University Graduate School of Medicine, North 15, West 7, Kita-ku, Sapporo 060-8638, Japan. email: nekozamurai@me.com. 